\title{
Current Density Distribution in Cylindrical Li-Ion Cells during Impedance Measurements
}

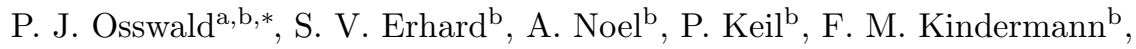 \\ H. Hoster ${ }^{\mathrm{a}, \mathrm{c}}$, A. Jossen ${ }^{\mathrm{b}}$ \\ ${ }^{a}$ TUM CREATE Ltd., Singapore \\ ${ }^{b}$ Institute for Electrical Energy Storage Technology (EES), Technical University of Munich \\ (TUM), Munich \\ ${ }^{c}$ Energy Lancaster, Lancaster University, Lancaster
}

\begin{abstract}
In this work, modified commercial cylindrical lithium-ion cells with multiple separate current tabs are used to analyze the influence of tab pattern, frequency and temperature on electrochemical impedance spectroscopy. In a first step, the effect of different current tab arrangements on the impedance spectra is analyzed and possible electrochemical causes are discussed. In a second step, one terminal is used to apply a sinusoidal current while the other terminals are used to monitor the local potential distribution at different positions along the electrodes of the cell. It is observed that the characteristic decay of the voltage amplitude along the electrode changes non-linearly with frequency, where highfrequent currents experience a stronger attenuation along the current collector than low-frequent currents.

In further experiments, the decay characteristic is controlled by the cell temperature, driven by the increasing resistance of the current collector and the enhanced kinetic and transport properties of the active material and electrolyte. Measurements indicate that the ac current distribution depends strongly on the frequency and the temperature. In this context, the challenges for electrochemical impedance spectroscopy as cell diagnostic technique for commercial cells are discussed.
\end{abstract}

\footnotetext{
* Corresponding author

Email address: pjo@tum.de (P. J. Osswald)
}

Preprint submitted to Journal of Power Sources

March 14, 2016 
Keywords: Lithium-ion battery, Impedance, current density distribution, local potential measurements, aging, cell design

\section{Introduction}

For more than a decade, batteries have become one of the main research objects to tackle the problem of air pollution caused by fossil fuels for transportation and energy production. Intense research and development effort has been devoted to lithium-ion batteries (LIB), as they were identified as one of the most promising solution. Nowadays, due to the steady increase in power density, safety and reliability, LIB are used not only for consumer electronics anymore, but also stationary electrical energy storage as well as electric and hybrid vehicles. As diverse as these applications are, as diverse are the different types of cells used for them. Most common designs are cylindrical cells, prismatic hard-case cells and pouch cells [1, 2, 3]. All of those cell types have in common that they can be monitored by electrochemical impedance spectroscopy (EIS) to determine their inner states [4]. The sensitivities of EIS measurements to the state of charge $(\mathrm{SOC})[5,6]$, state of health $(\mathrm{SOH})[7,8,9]$, cell temperature $[10,11]$ and short-term previous charge and discharge history [12] are discussed in literature. Since commercial cells have one single electrical terminal, EIS measurements for diagnostic issues face the drawback that different impedance contributions of different locations at the current collectors are superimposed at the terminal. On the contrary, studies of current density distributions at typical cycling conditions show an inhomogeneous operation along the electrodes $[13,14,15]$. The distribution of a sinusoidal current through the electrodes is not discussed in present literature. Thus, it can be assumed that an alternating current (ac) operation also leads to inhomogeneous current distribution through the electrode. As a consequence, EIS measurements would provide impedance data from undefined fractions of the electrodes. Especially in the context of monitoring aging in commercial cells, the impact of local inhomogeneities on the measured spectra have to be considered. This paper discusses experimental 
investigations of the current path evoked by an ac excitation current in dependence of different tab patterns, frequencies and temperature. Therefore, a commercial 26650 type lithium-ion cell with a lithium iron phosphate (LFP) cathode and a graphite anode was modified to allow for local measurements of the electrode potentials[15, 16]. The experimental details and testing scenarios are described, followed by the discussion of the experimental data involving both, the time and frequency domain.

\section{Experimental work}

In this work, measurements were performed on modified commercial 26650 cylindrical cells. The original cell has a nominal capacity of 2.5 Ah with LFP as cathode and graphite as anode material. Prior to modification, the cell has four positive and four negative current tabs, distributed equidistantly along the electrodes and welded together to the respective part of the cell casing. By modification, we have disconnected these welding joints and separated the single current tabs, allowing an individual access to each of the eight current tabs, as shown in Fig. 1 a). For more details on the modification process and cell parameters, please refer to our previous work [15]. Based on the tab arrangement $A_{m}$ and $C_{n}$, with $m, n \in\{1 ; 4\}$ used for the measurement, we define a terminal $T_{m n}$ with its respective terminal voltage $U_{T m n}$ and impedance $Z_{T m n}$, respectively. A cylindrical cell is used, with $T_{11}\left(A_{1}-C_{1}\right)$ as the outermost and $T_{44}\left(A_{4}-C_{4}\right)$ as the innermost terminal. To help visualizing the cell, Fig. 1 depicts a sketch of the modified cell (b) together with pictures of the current tabs of an opened cell at anode (c) and cathode side (d). After resealing, the modified cell can be operated outside of the glovebox. All tests were performed within the first 20 cycles. As shown in our previous work, modified cells age during cycling for 200 cycles similar to unmodified cells and show a capacity fade of $10 \%$ after 1000 cycles [15]. Therefore, we concluded that no electrochemical change will occur during our measurements.

To charge and discharge the cells, a BaSyTec CTS was used. To adjust the 

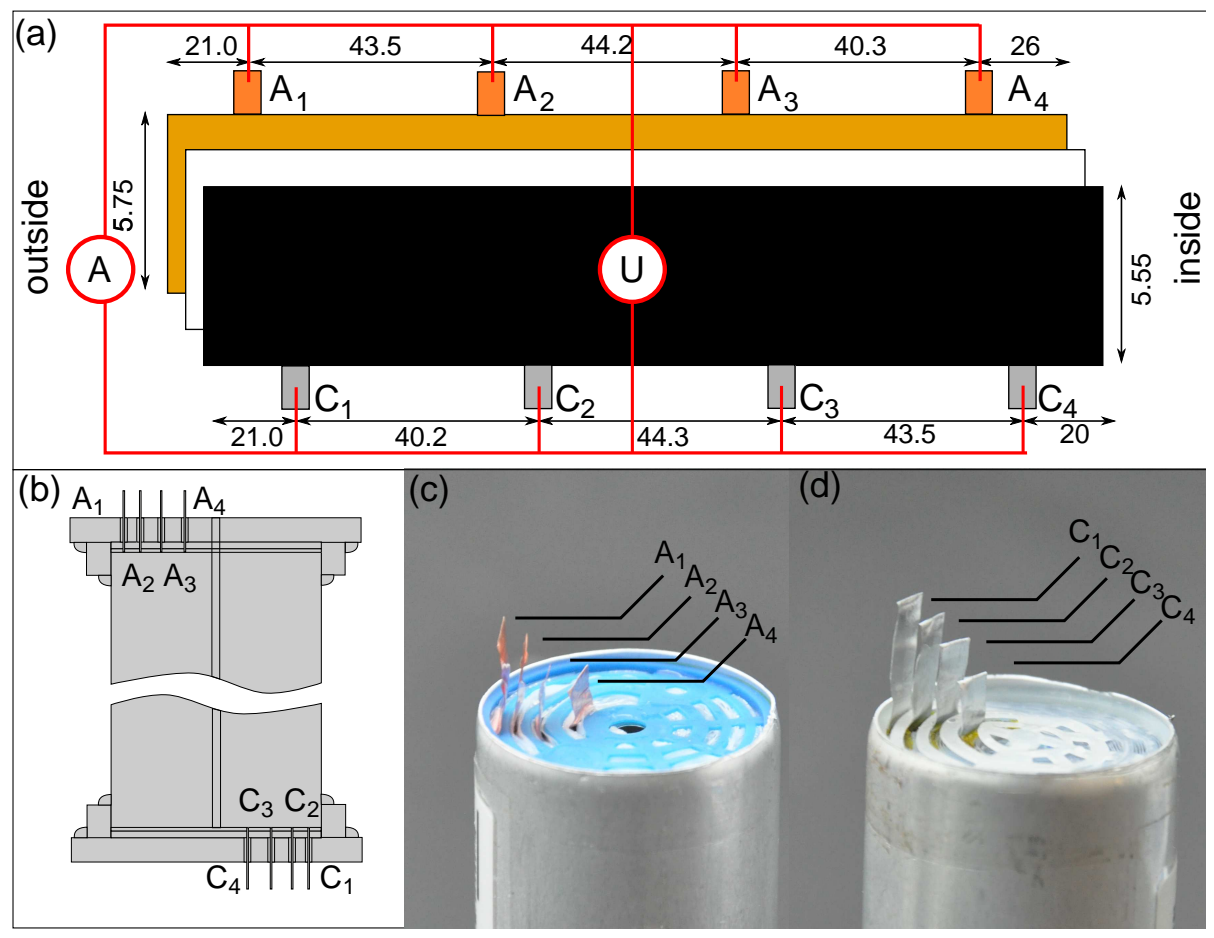

Figure 1: Drawing of the cell with dimensions in $\mathrm{cm}$ (a) and schematic cross-section after the modification process (b) [15]. The pictures show the opened cell with four current tabs on anode (c) and cathode (d). 
SOC of the cells, a current of $0.1 \mathrm{C}(250 \mathrm{~mA})$ was applied using two positive current tabs $\left(C_{1}\right.$ and $\left.C_{3}\right)$ and two negative current tabs $\left(A_{2}\right.$ and $\left.A_{4}\right)$. This asymmetrical operation mode is assumed to reduce local SOC inhomogeneities and, therefore, to reduce the time until the cell reaches an equilibrated state, a prerequisite for accurate impedance measurements [17, 18]. For impedance measurements, a Bio-Logic SAS VMP-3 has been used and different excitation currents between $30 \mathrm{~mA}$ and $200 \mathrm{~mA}$ were applied. The frequency ranges from $100 \mathrm{mHz}$ to $10 \mathrm{kHz}$ with 6 points per decade and 3 measurements per frequency.

During the galvanostatic control at one terminal, further potential measurements at the additional terminals are possible. For example, during the operation via terminal $T_{11}$, the terminal voltage $U_{T 22}, U_{T 33}$ and $U_{T 44}$ allow the observation of the potential drop along the current collector and the induced changes in the current density distribution with the resulting local SOC inhomogeneities. For the potential measurements between the supplementary tabs, additional channels of the Bio-Logic SAS VMP-3 were used with a sampling rate of up to $5 \mathrm{kHz}$. According to the product specification, a resolution of $5 \mu \mathrm{V}$ is achieved for the chosen voltage range.

The temperature of the cell was controlled with a Memmert IPP200 incubator and an ESPEC climate chamber. The cell was placed into the chamber $2 \mathrm{~h}$ prior to the measurement to allow for temperature adjustment, leading to a homogenous temperature distribution within the cell [19]. During the measurement, a short rest period of $5 \mathrm{~min}$ to $10 \mathrm{~min}$ was maintained, after the tab pattern for the excitation current was changed, to eliminate temperature variations.

\section{Results and Discussion}

The modification process allowed reliable and reproducible results with only negligible impact on the measurements within the time domain at constant currents [15]. To verify these measurements in the frequency domain, Fig. 2 compares the Nyquist plot of an unmodified cell with a modified cell, where 
all four current tabs on each electrode were connected, measured at $25^{\circ} \mathrm{C}$ and $50 \%$ SOC. The resulting spectra match well for the relevant frequencies between $100 \mathrm{mHz}$ and $10 \mathrm{kHz}$, except for a shift along the $Z^{\prime}$-axis. Due to the modification process, the connection to the cells are changed, resulted in additional contact resistances at the current tabs. The higher value for $Z^{\prime}$ is a sum of contact resistances, consisting of the newly added welding spots, the tab extensions as well as the soldered banana plugs to connect the cell and therefore causes a shift of the spectra along the $Z^{\prime}$-axis. This was further investigated by comparing the two modified cells used in this work. The measurements revealed nearly identical spectra over a wide frequency range, whereas minor differences in the high frequency region can be caused by the measurement equipment. Especially at very high frequencies even cable alignment might affect the inductive part. The shift along the $Z^{\prime}$-axis indicates the varying ohmic contact resistances which does not distort the measurements. For completeness, the data of both cells for different operations modes are presented in Fig. 8 in the appendix.

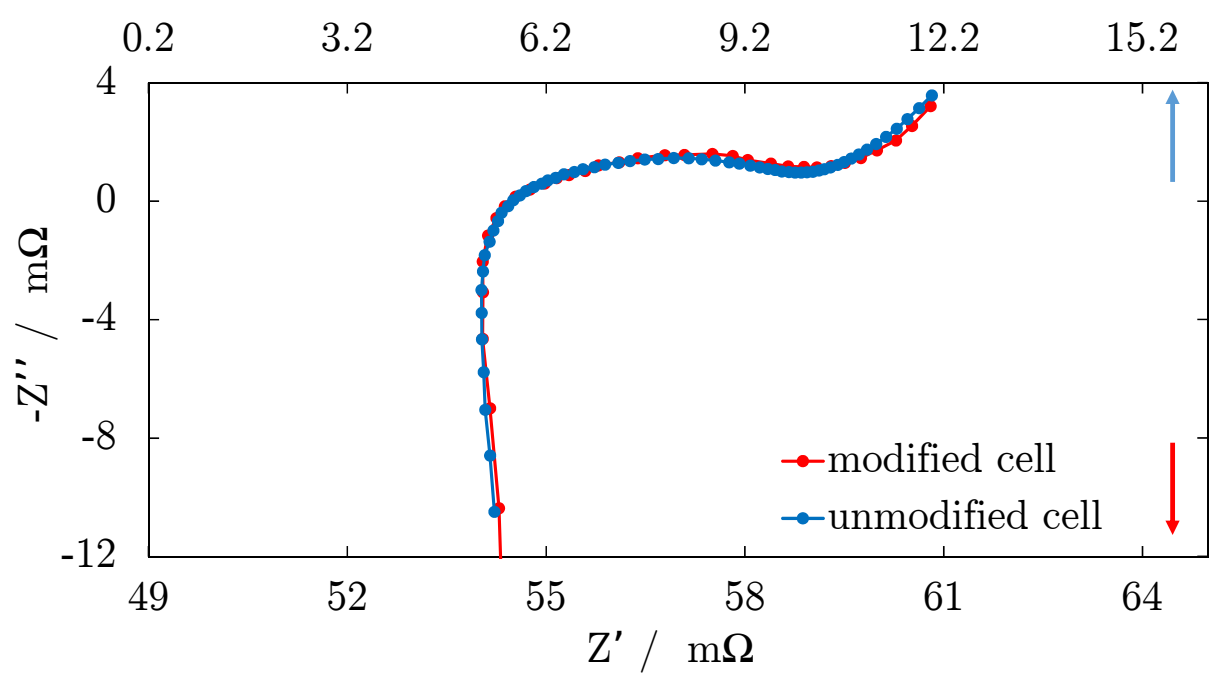

Figure 2: Nyquist plot of the cylindrical cell prior (blue) and post (red) modification process. For the measurement, all four tabs on each electrode were connected. Please note the usage of the second $Z^{\prime}$-axis to compensate the shift in $Z^{\prime}$ for the modified cell 


\subsection{Tab pattern}

Different current tab patterns were used to apply the sinusoidal current to the cell. Due to the multiple arrangement possibilities, the measurements were divided in three groups, namely $\mathrm{A}, \mathrm{B}$ and $\mathrm{C}$ as depicted in Fig. $3 \mathrm{a}-\mathrm{c}$ ). For group A, the particular opposite current tabs of anode and cathode were combined $\left(A_{x}-C_{x}\right)$ to allow measurements along the electrode from outside $\left(T_{11}\right)$ to inside $\left(T_{44}\right)$ the cell. Group B used diagonally arranged current tabs, where the positive current tab was combined with the next negative current tab closer to the cell center $\left(A_{x+1}-C_{x}\right)$. In the last group $\mathrm{C}$, the negative current tab $A_{1}$ was used throughout all measurements in combination with all four positive current tabs $\left(A_{1}-C_{x}\right)$. The additional contact resistances caused a distribution of the Nyquist plots along the $Z^{\prime}$-axis. For purpose of illustration, all spectra in the Nyquist plots in Fig. 3 were shifted along the $Z^{\prime}$-axis, defining the measurement at terminal $T_{11}$ as the reference value with $Z^{\prime}\left(Z^{\prime \prime}=0\right)=37.9 \mathrm{~m} \Omega .|Z|$ and $\phi$ where recalculated using the adjusted $Z^{\prime}$ values, compensating the influence of additional contact resistances. For comparison, the Nyquist plots and Bode diagrams with original data and the unadjusted $Z^{\prime}$ values are illustrated in the appendix (Fig. 8). The expected experimental error originating from the measurement equipment can be calculated to be less than $1 \%$ for an excitation current of $<50 \mathrm{~mA}$. The high reproducibility of the measurements for both modified cells leads to the assumptions, that further errors originating from the connection of the cell to the equipment are negligible compared to the measured effects.

\subsubsection{Group A}

In group A (Fig. 3 a)) the sinusoidal current is applied between the opposing current tabs, denoted as $A_{x}-C_{x}$. This leads to four different measurements with $A_{1}-C_{1}$ as the outermost and $A_{4}-C_{4}$ as the innermost tab configuration in the cylindrical cell.

The Nyquist plot shows a good agreement between the performed measurements in the high frequency region above $1000 \mathrm{~Hz}$. For frequencies below 


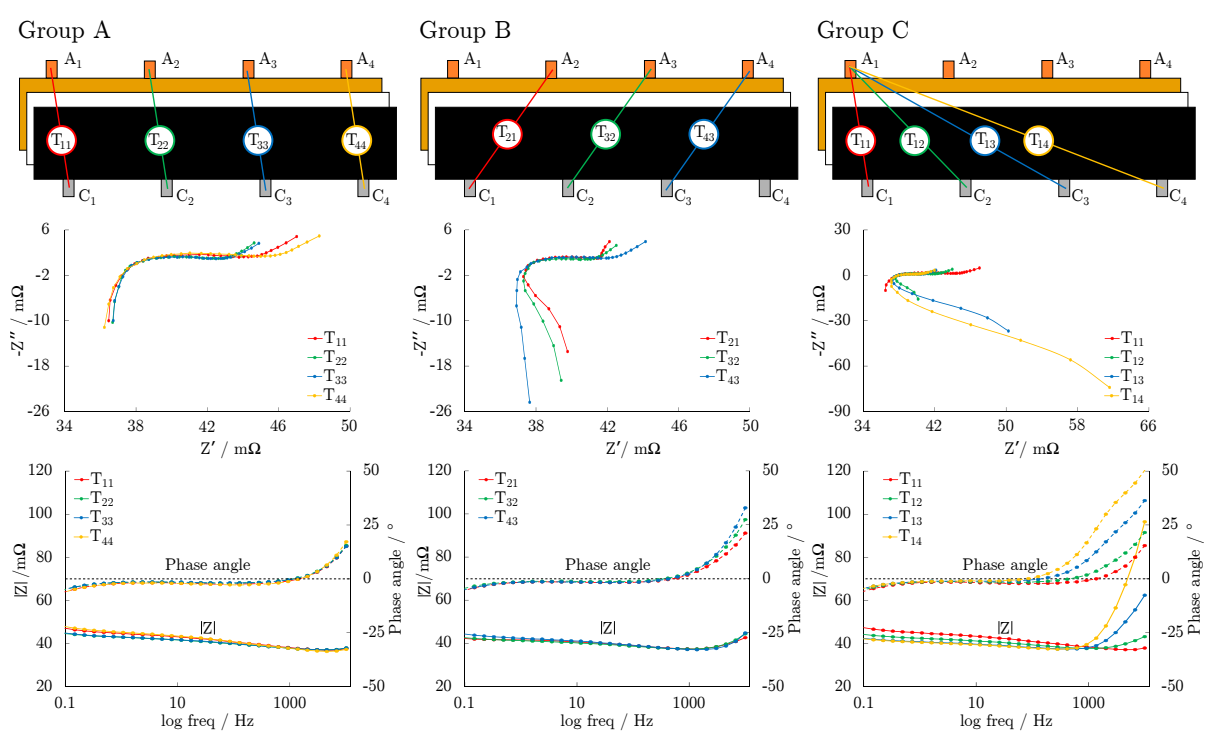

Figure 3: Different measurement sets, divided in group A, B and C based on tab pattern used for the impedance measurement and the resulting Nyquist and Bode plots. Please note that for the purpose of illustration, the data have been adjusted to a defined $Z^{\prime}$ value and that the axis labelling between the Nyquist plots varies to highlight the differences between the tab configurations. The unadjusted data are shown in the appendix 
$1000 \mathrm{~Hz}$, a significant increase of $Z^{\prime}$ is observed for the two measurements when terminals $T_{11}$ and $T_{44}$ were used in comparison to terminals $T_{22}$ and $T_{33}$. The Bode diagram indicates no significant differences in the phase angle between the measurements and the cell changes for all four measurements from inductive to capacitive behavior at nearly the same frequency of about $1350 \mathrm{~Hz}$. In the Bode diagram, $\left|Z_{T 11}\right|$ and $\left|Z_{T 44}\right|$ increase towards lower frequencies due to the higher values of $Z_{T 11}^{\prime}$ and $Z_{T 44}^{\prime}$.

In respect to the geometric design of the cell, the location of the terminals $T_{11}$ and $T_{44}$ exhibit similar properties in respect to their position on the current collector. The current tabs are located close to one end of the electrode with a length of 20 and $21 \mathrm{~cm}$, respectively, to one side and $140 \mathrm{~cm}$ to the other side of the positive electrode. However, terminal $T_{22}$ and $T_{33}$ are located closer to the middle of the electrode and possess approximately 60 and $100 \mathrm{~cm}$ of electrode material on the left and on the right side. This leads to the conclusion, that the current density within the cell is distributed asymmetrically and the potential drop along the current collector causes an increase in the resistance $Z^{\prime}$ for the terminals $T_{11}$ and $T_{44}$.

Although terminal $T_{22}$ and $T_{33}$ on one hand and terminal $T_{11}$ and $T_{44}$ on the other, show very similar behavior, the impedance $Z^{\prime}$ of the terminal closer to the cell center is higher than the other $\left(Z_{T 11}^{\prime}=47 \mathrm{~m} \Omega, Z_{T 44}^{\prime}=47.9 \mathrm{~m} \Omega\right.$ for $100 \mathrm{mHz}$ ). Frequencies below $10 \mathrm{~Hz}$ are assigned to the charge transfer and the diffusive behavior of the lithium ions in the bulk material. Since no temperature gradient is present in the cell and the SOC has been adjusted carefully to avoid any inhomogeneities, it is assumed that inhomogeneous kinetic properties of the active material or electrolyte cause the differences. Simulations on the stress evolution in cylindrical cells have been published by Nadimpalli et. al. [20] and the work of Waldmann et. al. [21] reported mechanical deformation of cylindrical cells after cycling, where a thermo-mechanical stress had caused the jelly roll to kink if no center pin in the cell was present. A center pin is a small steel rod, which is inserted during the manufacturing process when the electrode is wound. As the tested cell has a center pin, an increase of pressure towards the 
center of the cylindrical cell is assumed. Changes in bulk transport properties, higher impedances and lithium plating due to increasing pressure [22, 23, 24] and the relation between pressure, porosity and tortuosity of separators were reported in literature $[25,26]$.

As a consequence, we assume a reduced amount of electrolyte in the region close to terminal $T_{44}$ in comparison to the region close to terminal $T_{11}$. Less electrolyte and an increase in the lithium ion diffusion path due to a higher tortuosity in the separator could lead to a higher impedance, which becomes visible as increasing arc in the Nyquist plot. A change of active material thickness, in particular the double coated LFP electrode, caused by the smaller winding radius towards the cell center was also under consideration. It was calculated to be approximately 1.5\% thickness increase for the inner and $1.3 \%$ thickness decrease for the outer active material coating layer (coating thickness $d_{\text {electrode+ }}=63 \mu \mathrm{m}$, outer radius $r_{o}=12.5 \mathrm{~mm}$, inner radius $r_{i}=2 \mathrm{~mm}$ ). As this change is rather small, we assume that the effect on the kinetic properties of the active material is negligible. In summary, we observe a strong increase in impedance, if the excitation current was applied at terminals close to the end of the electrode due to an asymmetrical current density distribution $\left(Z_{T 11}, Z_{T 44}\right.$ $\left.>>Z_{T 22}, Z_{T 33}\right)$ and a smaller increase in impedance, if the cell geometry was the same but the current terminal was located closer to the cell center $\left(Z_{T 44}>\right.$ $Z_{T 11}$ and $\left.Z_{T 33}>Z_{T 22}\right)$ due to changes in the transport processes in the active material.

\subsubsection{Group B}

The results, suggesting a pressure increase towards the cell center, match very well with consecutive measurements of group B, presented in Fig. 3 b). In this operation mode, the excitation current was applied diagonally between the positive current tab $C_{x}$ and the negative current tab $A_{x+1}$. This allowed three possible ways to connect the tabs with a similar distance between the negative and the positive current tab of approximately $42 \mathrm{~cm}$. Since the measurements for $A_{x+1}-C_{x}$ show the same behavior as the measurements of $A_{x}-C_{x+1}$, 
only one data set is shown. For frequencies above $1500 \mathrm{~Hz}$, an increase of $Z^{\prime \prime}$ and a decrease of $Z^{\prime}$ is observed in the Nyquist plot and an increasing phase angle $\phi$ occurs in the Bode diagram. For lower frequencies, $\phi$ converges to the same value for the three measurements. While $Z^{\prime \prime}$ converges as well, $Z^{\prime}$ increases significantly for $A_{4}-C_{3}$ compared to $A_{2}-C_{1}$. This supports the aforementioned suggestion of a pressure effectuated increase in the lithium ion diffusion path in the liquid phase towards the cell center.

For changes in the high frequency region, the influence of the cell geometry has to be considered. The inductive behavior of the cell in this frequency region is attributed to the windings of the electrodes and connections inside the cell [27]. The distance between two current tabs remains nearly the same, but the increasing number of included windings of the jelly roll contributes to an increase. The number of windings $\Theta$ between the current tabs can be calculated with the adapted equation for the Archimedes' Spiral for cylindrical cells [28, 29]

$$
\Theta(\xi) \approx \frac{-r_{0}+\sqrt{r_{0}^{2}+\frac{D \xi}{\pi}}}{\frac{D \xi}{2 \pi}}
$$

whereas $r_{0}=1.5 \mathrm{~mm}$ is the radius of the center pin, $\xi$ is the distance of the tab to the cell center according to Fig. 1 a) and the thickness of the electrode stack $D=0.279 \mathrm{~mm}$ calculated by

$$
D=2 \cdot\left(d_{\text {separator }}+d_{\text {electrode }+}+d_{\text {electrode- }}\right)+d_{c c+}+d_{c c-}
$$

The number of electrode windings between the respective tabs was calculated to be 6.5 windings between $C_{1}$ and $A_{2}, 8.5$ between $C_{2}$ and $A_{3}$ and 12.5 between $C_{3}$ to $A_{4}$. The half winding originates from the cell design as displayed in Fig. 1 b), where the current tabs of the anode are shifted by half a winding compared to the current tabs of the cathode. This suggests an inductive contribution of the current collector, depending on the average radius of the cell segment and therefore the number of traversed windings. 


\subsubsection{Group $C$}

Group C consists of four set-ups, where the negative current tab $A_{1}$ is used for all measurements and one of the four positive current tabs $C_{1}, C_{2}, C_{3}$ and $C_{4}$ is used as the counterpart, denoted as $A_{1}-C_{x}$. As the mirrored measurements $A_{x}-C_{1}$ lead to the same results, only one data set is shown.

Table 1: Position of cathode tab $x$ with the respective number of windings, the distance between cathode and anode tab and the transition frequency with $\phi=0$

\begin{tabular}{rrrr}
$C_{x}$ & $N_{w}$ & Tab distance $/ \mathrm{cm}$ & $f(\phi=0) / \mathrm{Hz}$ \\
\hline 1 & 0.5 & 3.6 & 1400 \\
2 & 6.5 & 43.8 & 500 \\
3 & 14.5 & 88.1 & 160 \\
4 & 26.5 & 131.6 & 80
\end{tabular}

The distances and number of windings between the current tabs were calculated and are stated in Tab. 1. The Nyquist plot in Fig. 3 c) exhibits a strong correlation of $Z^{\prime \prime}$ as well as $Z^{\prime}$ with the distance and number of electrode windings between the current tabs. At $10 \mathrm{kHz}, Z_{14}^{\prime}$ increases by nearly $70 \%$, compared to $Z_{11}^{\prime}$ at the same frequency, whereas $Z_{14}^{\prime \prime}$ increased by the factor of $7.4(10 \mathrm{~m} \Omega$ to $74.2 \mathrm{~m} \Omega)$ compared to $Z_{11}^{\prime \prime}$. In agreement with the previous measurements in section 3.1.2, an increase of $Z^{\prime \prime}$ and therefore a more inductive behavior of the cell at high frequencies is observed with increasing number of utilized electrode windings. As illustrated in the Bode diagram, the cell's transition from inductive to capacitive behavior is shifted significantly to lower frequencies with increasing tab distance and number of windings. For tab configuration $A_{1}-C_{1}, \phi$ becomes negative for frequencies lower $1400 \mathrm{~Hz}$. With increasing tab distance, this characteristic frequency reduces to $500 \mathrm{~Hz}$ for $A_{1}-C_{2}, 160 \mathrm{~Hz}$ for $A_{1}-C_{3}$ and $80 \mathrm{~Hz}$ for $A_{1}-C_{4}$. For lower frequencies, the phase angle as well as $Z^{\prime \prime}$ become similar for all four measurements, but an increase of $Z^{\prime}$ is measured. In this case, the shorter distances between the tabs and the asymmetrical current density distribution result in an increase of $Z^{\prime}$. 


\subsection{Frequency dependency}

For the measurements presented in Fig. 4, an excitation current was applied at terminal $T_{11}$ and the voltage responses were sensed at terminals $T_{11}, T_{22}$, $T_{33}$ and $T_{44}$, measured with the second modified cell. Due to the decay of the current density along the current collector, the magnitude $|Z|$ (Fig. 4 a)) is smaller with increasing distance to the current tab. The phase angle $\phi$ (Fig. $4 \mathrm{~b})$ ) shows a higher inductive contribution for measurements towards the cell center and the transition frequency from inductive to capacitive behavior is shifted significantly.
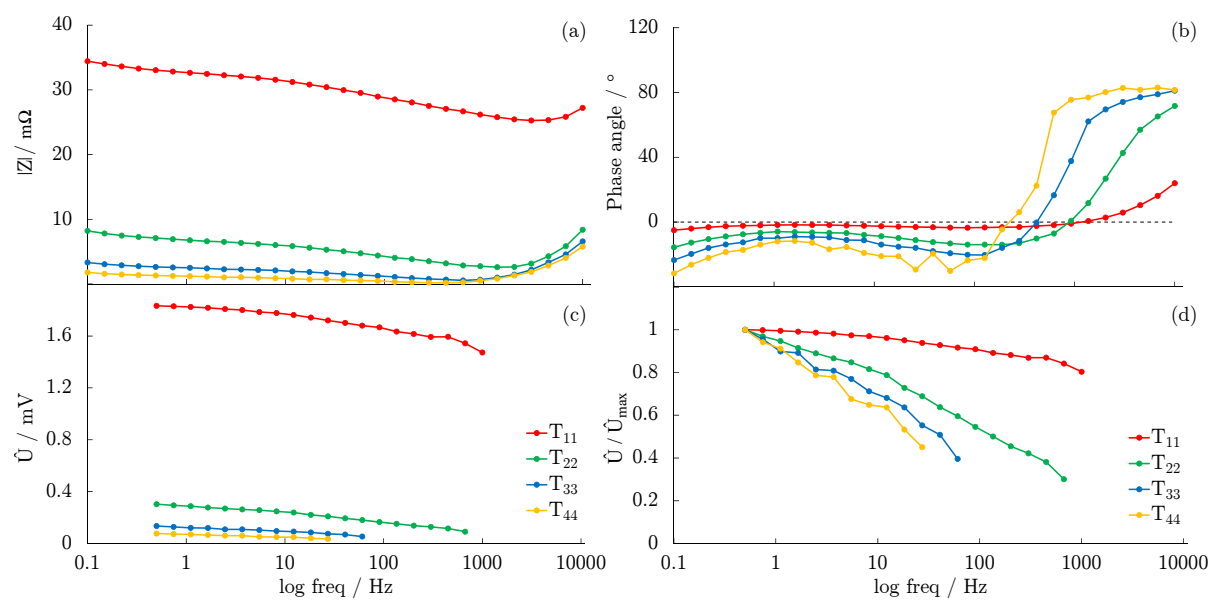

Figure 4: $|Z|$ (a), phase angle $\phi(\mathrm{b})$, voltage amplitude $\hat{U}$ (c) and the relative change of voltage amplitude $\hat{U} / \hat{U}_{\max }$ (d) in dependence of the frequency of the applied sinusoidal current

Since the interpretation of an impedance spectrum might be misleading, when excitation and sensing terminal are separated, we performed an additional measurement, sensing the potential within time domain. The amplitude $\hat{U}$ is plotted in Fig. $4 \mathrm{c}$ ). The potential measurement indicates the reliability of the impedance measurement within the detectable frequency range. $\hat{U}_{11}$ depicts the largest value, as the excitation current is applied at terminal $T_{11}$, similar to the values for magnitude $\left|Z_{T 11}\right|$ in the plot above. Both values decrease with increasing distance to the current tab. The magnitudes $|Z|$ and amplitudes $\hat{U}$ at all terminals decrease with increasing frequency, but in a non-linear manner. 
This is highlighted in Fig. 4 d), by showing normalized values. Thereby, $\hat{U}_{\max }$ corresponds to the value of $\hat{U}$ at the lowest frequency. These measurements suggest that the current density distribution along the current collector changes non-uniformly depending on the applied frequency, measured as a higher attenuation of the voltage response for higher frequencies.

As discussed in literature $[4,30,31,32]$, an impedance spectrum senses different electrochemical processes with distributed time constants. For the high frequency region above $1000 \mathrm{~Hz}$, the major contributions occur due to the measurement cable and terminal set-up, the cell geometry as well as polarization losses originating from contact resistances between the coating of the active material and the current collector. The behavior for mid-range frequency between $1000 \mathrm{~Hz}$ and $10 \mathrm{~Hz}$ is dominated by the charge transfer reaction between electrode and electrolyte and lithium ion diffusion within electrolyte, while the solid state diffusion in the active material defines the spectra for frequencies below $10 \mathrm{~Hz}[33,34]$. We can conclude that the current density distribution within the cell depends on the applied frequency due to different sources of impedance, corresponding to different electrochemical processes. As a consequence, it is possible to shift the current density distribution as these processes are highly sensitive to the cell temperature as discussed in the following section.

\subsection{Temperature dependency}

In Fig. 5, the Nyquist plot (a) and the Bode diagram (b) are displayed for different excitation currents (50 mA, $100 \mathrm{~mA}$ and $200 \mathrm{~mA}$ ) as well as different temperatures $\left(10{ }^{\circ} \mathrm{C}, 25^{\circ} \mathrm{C}\right.$ and $\left.40{ }^{\circ} \mathrm{C}\right)$. Similar to the measurements in the previous section, all measurements were performed using terminal $T_{11}$. No current dependency of the impedance measurement was observed, as the linearity of the system was still valid for the maximum excitation current of $200 \mathrm{~mA}$ [35].

In Fig. 5. with increasing temperature, a significant reduction of $Z^{\prime}$ can be observed for frequencies below $1000 \mathrm{~Hz}$, attributable to enhanced kinetic properties such as lower electrolyte resistance, higher diffusion coefficients in the active material and a lower charge transfer resistance at the solid electrolyte 

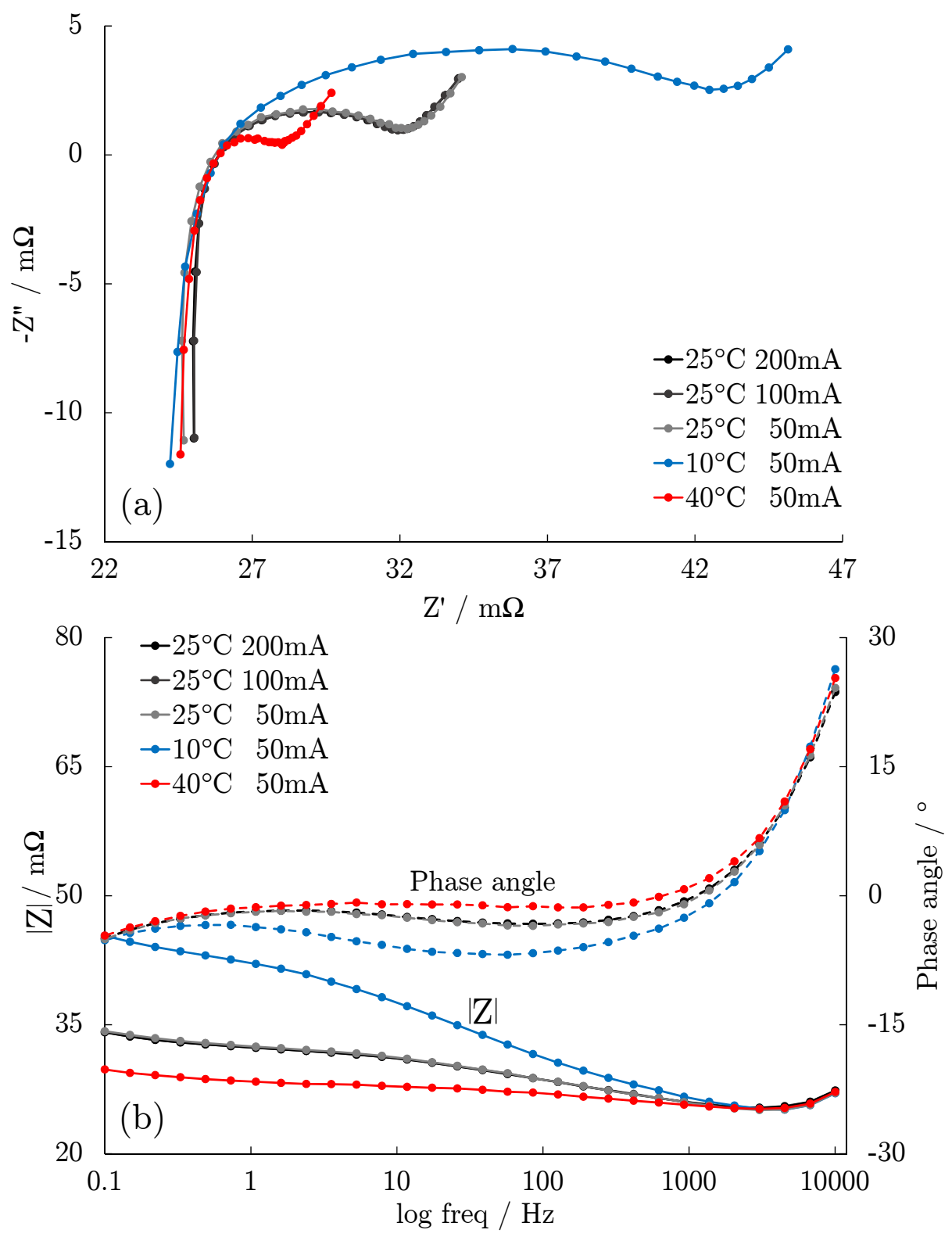

Figure 5: Nyquist plot (a) and Bode diagram (b) recorded at $25^{\circ} \mathrm{C}$ with different ac excitation currents $(50,100$ and $200 \mathrm{~mA})$ as well as at different temperatures $\left(10,25\right.$ and $\left.40^{\circ} \mathrm{C}\right)$. 
interface [36, 37]. The Bode diagram in Fig. 5 b) illustrates the temperature independence of the impedance for frequencies above $2000 \mathrm{~Hz}$, with an identical phase angle $\phi$ and magnitude $|Z|$. With decreasing frequency, cell properties change from inductive to capacitive behavior $(\phi<0)$, whereas the cell at $10^{\circ} \mathrm{C}$ changes at higher frequencies compared to the cells at 25 and $40^{\circ} \mathrm{C}$. The phase angle decreases with higher temperature throughout the measured frequency range but all values start merging for frequencies below $1 \mathrm{~Hz}$. In addition to the terminal voltage $U_{T 11}$, terminal voltages $U_{T 22}, U_{T 33}$ and $U_{T 44}$ were recorded during the impedance measurement. Fig. 6 illustrates the voltage responses, measured at the different locations along the electrodes for $10{ }^{\circ} \mathrm{C}(\mathrm{a}), 25{ }^{\circ} \mathrm{C}$ (b) and $40{ }^{\circ} \mathrm{C}$ (c) for 240 and $167 \mathrm{mHz}$. For a clearer distinction, the terminal voltages were separated by $3 \mathrm{mV}$ along the y-axis.

These exemplary frequencies allow for an insight on the distribution of the excitation current along the electrode. They show the voltage amplitude attenuation along the current collector with a strong temperature dependency. For the last frequency applied $(167 \mathrm{mHz})$, the voltage amplitudes at the different terminals were calculated and plotted in Fig. 7 versus the distance to the current carrying tab, where the excitation current was applied. At $10{ }^{\circ} \mathrm{C}$, a voltage amplitude of $2.33 \mathrm{mV}$ is measured at terminal $T_{11}$ decreasing to $0.45 \mathrm{mV}$ at terminal $T_{44}$. A temperature increase to $40^{\circ} \mathrm{C}$ leads to a reduced voltage amplitude $\hat{U}_{T 11}=1.54 \mathrm{mV}$ at terminal $T_{11}$, decaying to $0.038 \mathrm{mV}$ at terminal $T_{44}$. Normalizing the curves in Fig. 7 a) to $\hat{U} / \hat{U}_{\max }$ (dotted lines) visualizes the strong non-linear temperature dependency of the voltage decay along the current collector, where an increase in temperature leads to a significant higher attenuation.

The positive temperature coefficients of resistance for aluminum $\left(\alpha_{A l}=\right.$ $\left.4 \cdot 10^{-3} \mathrm{~K}^{-1}\right)$ and copper $\left(\alpha_{C u}=3.93 \cdot 10^{-3} \mathrm{~K}^{-1}\right)$ lead to an increase in the resistance at higher temperatures. For the temperature range of $\Delta T=30^{\circ} \mathrm{C}$, the resistance of each current collector differs by approximately $12 \%$, which will lead to a stronger voltage attenuation along the current collector. This effect is amplified by the enhancements in kinetics and mass transport, which 


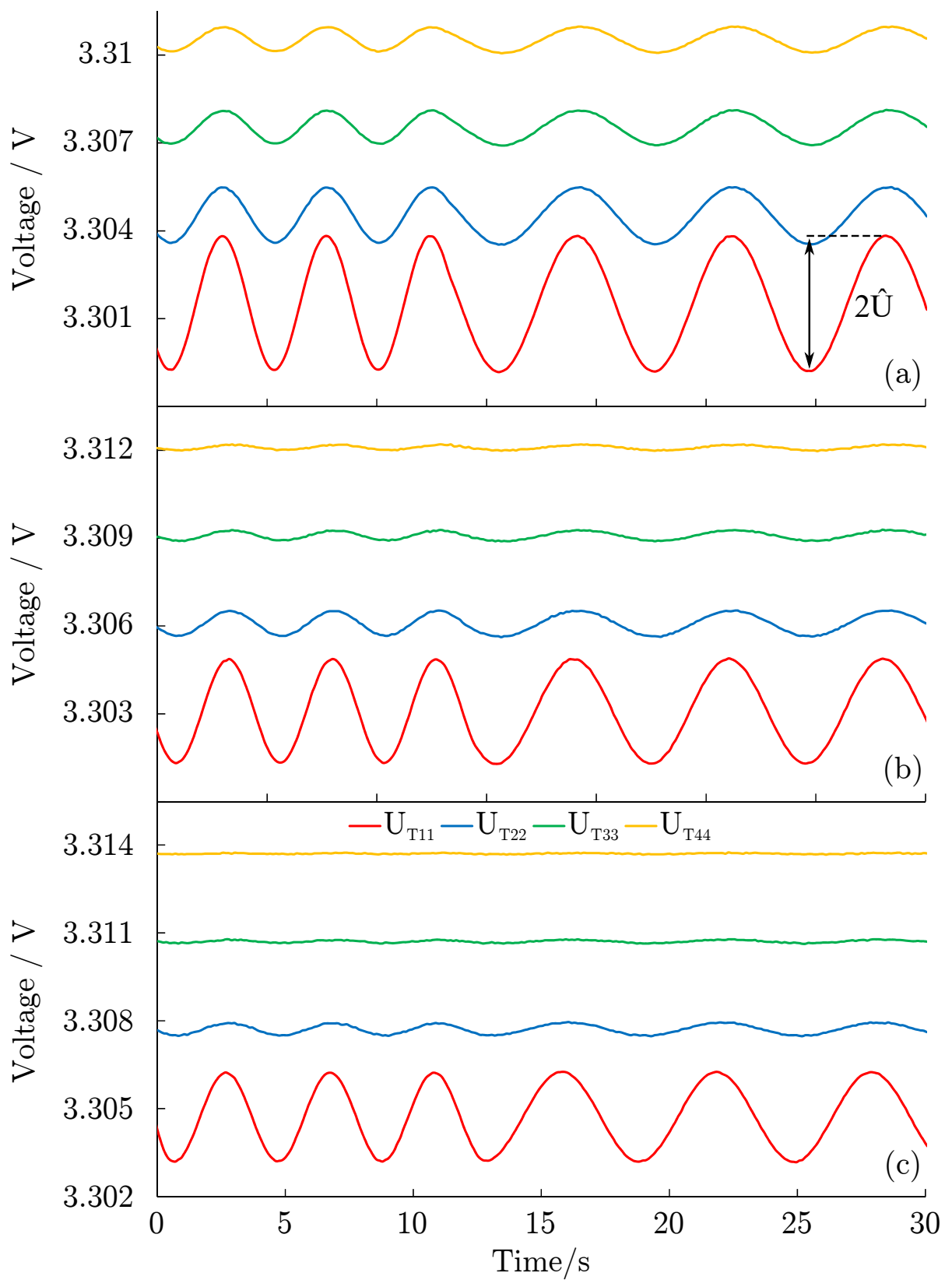

Figure 6: Cell's voltage response to a current excitation of $50 \mathrm{~mA}$ with a frequency of 240 and $167 \mathrm{mHz}$ at terminal $T_{11}$ at different positions for $10^{\circ} \mathrm{C}$ (a), $25^{\circ} \mathrm{C}$ (b) and $40{ }^{\circ} \mathrm{C}$ (c) (voltages were separated by $3 \mathrm{mV}$ for purpose of illustration) 
were reported to double (ionic conductivity in electrolyte [38]) or increase by the factor of 20 (lithium ion diffusion coefficient in LFP [39]) for an increase from $10^{\circ} \mathrm{C}$ to $40^{\circ} \mathrm{C}$. Thus, we suppose that enhanced kinetic and transport properties of the cell dominate the voltage attenuation, in comparison to the small effect of the decreasing current collector conductivity.

To validate the significance of the previous results, the linearity of the system was examined. As comparison, Fig. 7 b) illustrates the voltage amplitude for different excitation currents $(50,100,200 \mathrm{~mA}$; solid lines) at a constant temperature of $25^{\circ} \mathrm{C}$. Plotted as $\hat{U} / \hat{U}_{\max }$ (dashed lines), the same attenuation along the current collector for the different excitation currents is observed, indicating a proportional relation between voltage amplitude and excitation current, where doubling the current leads to a doubling of the voltage amplitude at all positions along the electrode.

\section{Conclusion}

In this work, we presented impedance measurements on modified commercial 26650 type lithium-ion cells. The modification allowed for applying any tab pattern to four cathode and four anode tabs, which are distributed nearly symmetrically and equidistantly along the electrodes. The focus of our study was set on ac current density distribution along the electrodes under variation of tab pattern, frequency and temperature.

The tab pattern variation demonstrated two major effects. On one hand, the low frequency impedance is higher for the innermost terminal $T_{44}$. Based on published literature, a pressure increase towards the cell center was found to be a reasonable explanation. On the other hand, the inductive high frequency behavior clearly reveals a dependency on involved cell windings. The cell inductance depends on the electrode length and radius of enclosed windings.

According to the superposition of separate impedance contributions, the frequency is a major variable when it comes to the current path through the cell. It can be observed that the signal path along the current collector and through 


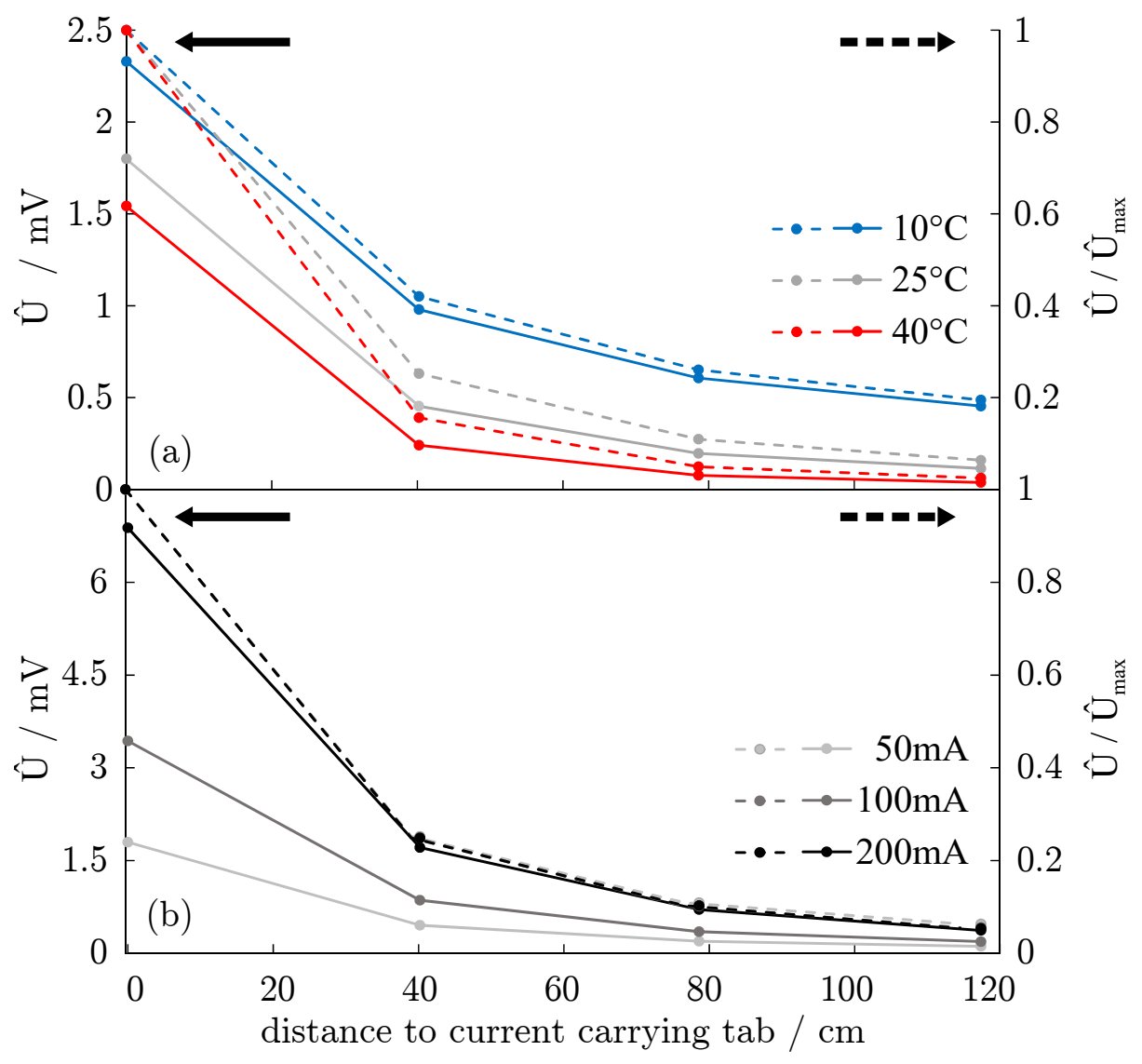

Figure 7: Amplitude of the voltage response $(f=167 \mathrm{mHz})$ at different temperatures for an ac excitation current with a frequency of $50 \mathrm{~mA}$ and (a) and for different ac excitation currents (b) at $25^{\circ} \mathrm{C}$ 
the electrochemically active layer is shifted by a variation in the excitation signal frequency. Higher frequencies tend to experience more attenuation, whereas lower frequencies have a wider spread along the current collectors.

Further, this inhomogeneous current path was highlighted by temperature variation. An increase in temperature increases the current collectors' resistivity, whereas the electrochemical active layer impedance will tend to lower values due to enhanced kinetics and lithium ion transport. We analyzed this correlation at three temperatures, 10,25 and $40{ }^{\circ} \mathrm{C}$ and detected a more pronounced gradient in ac current density distribution along the electrode as the temperature increased.

As EIS is one of the most common techniques for cell diagnostics, such as inner resistance measurements, estimation of $\mathrm{SOC}$ as well as $\mathrm{SOH}$, our findings might imply certain limitations to its interpretability when applied to commercial cells. In general, it is questionable, how a cell design overshadows the active material impedance by superimposing local inhomogeneities, such as increased passivation layers due to lithium plating or advanced aging. By applying the method of local potentials to single layered cells, we want to focus on that overshadowing in future work.

\section{Acknowledgment}

The presented work was supported by the Singapore National Research Foundation (NRF) through its Campus for Research Excellence and Technological Enterprise (CREATE) program, the Bavarian Ministry of Economic Affairs and Media, Energy and Technology under the auspices of the EEBatt project and the German Federal Ministry of Education and Research (funding reference number 03X4633A)

\section{Appendix}


Comparison between modified cell 1 and modified cell 2
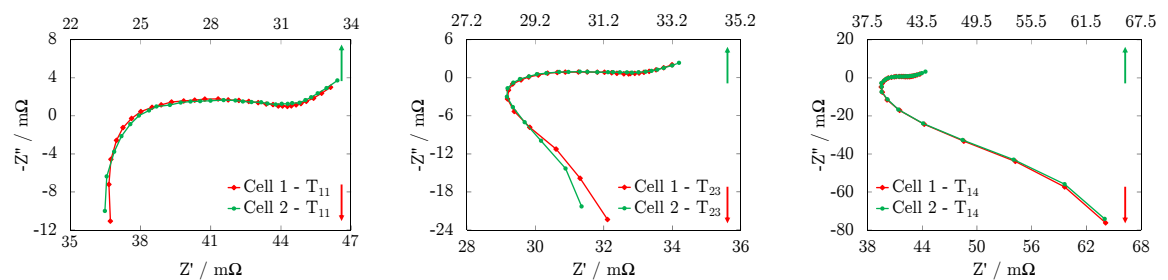

Original, unadjusted data of Figure 3.
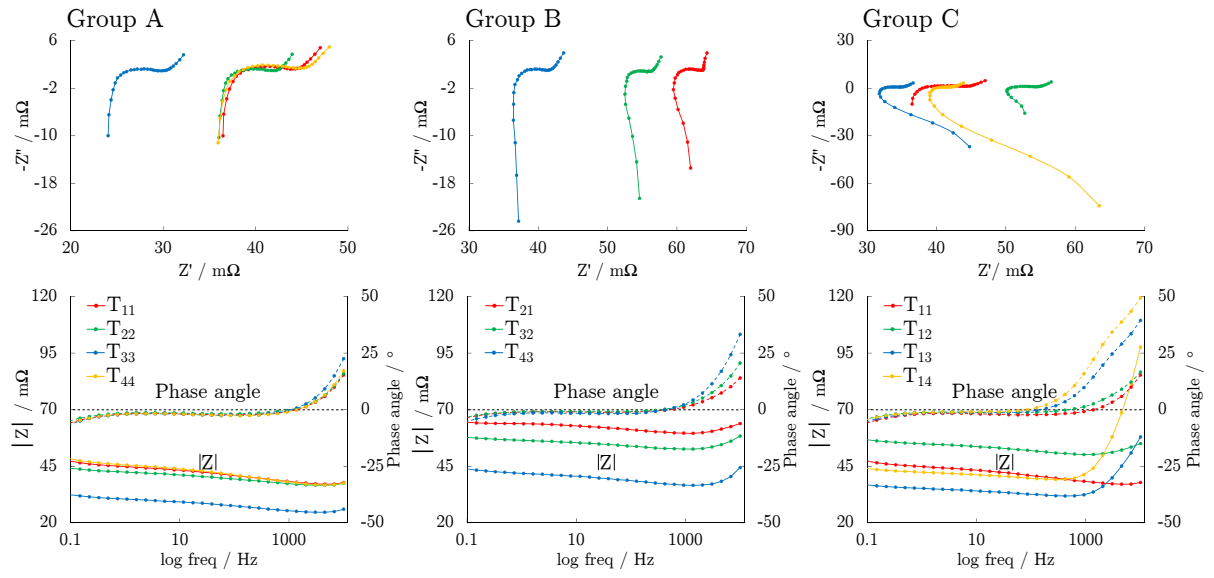

Figure 8: The comparison between the two modified test cells used in this work as well as the Nyquist plot and Bode diagram as presented in Fig 3 with the original, unadjusted data, measured with modified cell 1. For purpose of illustration, please note that the axis labelling of the Nyquist plots varies 
[1] M. Armand, J.-M. Tarascon, Building better batteries., Nature 451 (7179) (2008) 652-657.

[2] B. Scrosati, J. Garche, Lithium batteries: Status, prospects and future, Journal of Power Sources 195 (9) (2010) 2419-2430.

[3] B. Dunn, H. Kamath, J.-M. Tarascon, Electrical Energy Storage for the Grid: A Battery of Choices, Science 334 (6058) (2011) 928-935.

[4] J. Vetter, P. Novák, M. Wagner, C. Veit, K.-C. Möller, J. Besenhard, M. Winter, M. Wohlfahrt-Mehrens, C. Vogler, A. Hammouche, Ageing mechanisms in lithium-ion batteries, Journal of Power Sources 147 (12) (2005) 269-281.

[5] D. Aurbach, Common Electroanalytical Behavior of Li Intercalation Processes into Graphite and Transition Metal Oxides, Journal of The Electrochemical Society 145 (9) (1998) 3024.

[6] W. Waag, S. Käbitz, D. U. Sauer, Experimental investigation of the lithium-ion battery impedance characteristic at various conditions and aging states and its influence on the application, Applied Energy 102 (2013) 885-897.

[7] K. Amine, C. Chen, J. Liu, M. Hammond, A. Jansen, D. Dees, I. Bloom, D. Vissers, G. Henriksen, Factors responsible for impedance rise in high power lithium ion batteries, Journal of Power Sources 97-98 (2001) 684-687.

[8] U. Tröltzsch, O. Kanoun, H.-R. Tränkler, Characterizing aging effects of lithium ion batteries by impedance spectroscopy, Electrochimica Acta 51 (8-9) (2006) 1664-1672.

[9] M. Kassem, J. Bernard, R. Revel, S. Pélissier, F. Duclaud, C. Delacourt, Calendar aging of a graphite/LiFePO4 cell, Journal of Power Sources 208 (2012) 296-305. 
[10] J. B. Robinson, J. a. Darr, D. S. Eastwood, G. Hinds, P. D. Lee, P. R. Shearing, O. O. Taiwo, D. J. Brett, Non-uniform temperature distribution in Li-ion batteries during discharge A combined thermal imaging, X-ray micro-tomography and electrochemical impedance approach, Journal of Power Sources 252 (2014) 51-57.

[11] R. R. Richardson, P. T. Ireland, D. a. Howey, Battery internal temperature estimation by combined impedance and surface temperature measurement, Journal of Power Sources 265 (2014) 254-261.

[12] D. Andre, M. Meiler, K. Steiner, H. Walz, T. Soczka-Guth, D. U. Sauer, C. Wimmer, T. Soczka-Guth, D. U. Sauer, Characterization of high-power lithium-ion batteries by electrochemical impedance spectroscopy. I. Experimental investigation, Journal of Power Sources 196 (12) (2011) 5334-5341.

[13] M. Fleckenstein, O. Bohlen, M. A. Roscher, B. Bäker, Current density and state of charge inhomogeneities in Li-ion battery cells with LiFePO4 as cathode material due to temperature gradients, Journal of Power Sources 196 (10) (2011) 4769-4778.

[14] G. Zhang, C. E. Shaffer, C.-Y. Wang, C. D. Rahn, In-Situ Measurement of Current Distribution in a Li-Ion Cell, Journal of the Electrochemical Society 160 (4) (2013) A610-A615.

[15] P. J. Osswald, S. V. Erhard, J. Wilhelm, H. E. Hoster, A. Jossen, Simulation and Measurement of Local Potentials of Modified Commercial Cylindrical Cells, Journal of The Electrochemical Society 162 (10) (2015) A2099-A2105.

[16] S. V. Erhard, P. J. Osswald, J. Wilhelm, A. Rheinfeld, S. Kosch, A. Jossen, Simulation and Measurement of Local Potentials of Modified Commercial Cylindrical Cells, Journal of The Electrochemical Society 162 (14) (2015) A2707-A2719. 
[17] F. M. Kindermann, A. Noel, S. V. Erhard, A. Jossen, Long-term equalization effects in Li-ion batteries due to local state of charge inhomogeneities and their impact on impedance measurements, Electrochimica Acta 185 (2015) 107-116.

[18] A. Barai, G. H. Chouchelamane, Y. Guo, A. Mcgordon, P. Jennings, A study on the impact of lithium-ion cell relaxation on electrochemical impedance spectroscopy, Journal of Power Sources 280 (2015) 74-80.

[19] P. J. Osswald, M. D. Rosario, J. Garche, A. Jossen, H. E. Hoster, Fast and Accurate Measurement of Entropy Profiles of Commercial Lithium-Ion Cells, Electrochimica Acta 177 (2015) 270-276.

[20] S. P. Nadimpalli, V. A. Sethuraman, D. P. Abraham, A. F. Bower, P. R. Guduru, Stress Evolution in Lithium-Ion Composite Electrodes during Electrochemical Cycling and Resulting Internal Pressures on the Cell Casing, Journal of The Electrochemical Society 162 (14) (2015) A2656-A2663.

[21] T. Waldmann, S. Gorse, T. Samtleben, G. Schneider, V. Knoblauch, M. Wohlfahrt-Mehrens, A Mechanical Aging Mechanism in LithiumIon Batteries, Journal of the Electrochemical Society 161 (10) (2014) A1742-A1747.

[22] J. S. Gnanaraj, Y. S. Cohen, M. D. Levi, D. Aurbach, The effect of pressure on the electroanalytical response of graphite anodes and $\mathrm{Li}$ $\mathrm{CoO} 2$ cathodes for Li-ion batteries, Journal of Electroanalytical Chemistry $516(1-2)(2001)$ 89-102.

[23] T. C. Bach, S. F. Schuster, E. Fleder, J. Mller, M. J. Brand, H. Lorrmann, A. Jossen, G. Sextl, Nonlinear aging of cylindrical lithium-ion cells linked to heterogeneous compression, Journal of Energy Storage (2016) in press. 
[24] A. Barai, Y. Guo, A. McGordon, P. Jennings, A study of the effects of external pressure on the electrical performance of a lithium-ion pouch cell, in: Connected Vehicles and Expo (ICCVE), 2013 International Conference on, 2013, pp. 295-299.

[25] G. Venugopal, J. Moore, J. Howard, S. Pendalwar, Characterization of microporous separators for lithium-ion batteries, Journal of Power Sources 77 (1) (1999) 34-41.

[26] J. Cannarella, C. B. Arnold, Ion transport restriction in mechanically strained separator membranes, Journal of Power Sources 226 (2013) $149-155$.

[27] R. Mingant, J. Bernard, V. Sauvant-Moynot, A. Delaille, S. Mailley, J.-L. Hognon, F. Huet, EIS Measurements for Determining the SoC and SoH of Li-Ion Batteries, ECS Transactions 33 (2011) 41-53.

[28] E. W. Weisstein, "Archimedes' Spiral." From MathWorld-A Wolfram Web Resource, http://mathworld.wolfram.com/ArchimedesSpiral.html (September 2015).

URL http://mathworld.wolfram.com/ArchimedesSpiral.html

[29] M. Guo, R. E. White, Mathematical model for a spirally-wound lithium-ion cell, Journal of Power Sources 250 (2014) 220-235.

[30] M. D. Levi, K. Gamolsky, D. Aurbach, U. Heider, R. Oesten, On electrochemical impedance measurements of LixCo0.2Ni0.8O2 and LixNiO2 intercalation electrodes, Electrochimica Acta 45 (2000) 17811789.

[31] A. Jossen, Fundamentals of battery dynamics, Journal of Power Sources 154 (2) (2006) 530-538. 
[32] J. P. Schmidt, T. Chrobak, M. Ender, J. Illig, D. Klotz, E. IversTiffée, Studies on LiFePO4 as cathode material using impedance spectroscopy, Journal of Power Sources 196 (12) (2011) 5349-5355.

[33] J. Illig, M. Ender, T. Chrobak, J. P. Schmidt, D. Klotz, E. Ivers-Tiffee, Separation of Charge Transfer and Contact Resistance in LiFePO4Cathodes by Impedance Modeling, Journal of the Electrochemical Society 159 (7) (2012) A952-A960.

[34] J. Illig, J. P. Schmidt, M. Weiss, A. Weber, E. Ivers-Tiffée, Understanding the impedance spectrum of 18650 LiFePO4-cells, Journal of Power Sources 239 (2013) 670-679.

[35] E. Barsoukov, J. R. Macdonald, Impedance spectroscopy: theory, experiment, and applications, John Wiley \& Sons, 2005.

[36] A. N. Jansen, D. W. Dees, D. P. Abraham, K. Amine, G. L. Henriksen, Low-temperature study of lithium-ion cells using a LiySn microreference electrode, Journal of Power Sources 174 (2) (2007) 373-379.

[37] D. Y. W. Yu, C. Fietzek, W. Weydanz, K. Donoue, T. Inoue, H. Kurokawa, S. Fujitani, Study of LiFePO4 by Cyclic Voltammetry, Journal of The Electrochemical Society 154 (4) (2007) A253.

[38] D. M. Bernardi, J.-Y. Go, Analysis of pulse and relaxation behavior in lithium-ion batteries, Journal of Power Sources 196 (1) (2011) 412-427.

[39] Y.-R. Zhu, Y. Xie, R.-S. Zhu, J. Shu, L.-J. Jiang, H.-B. Qiao, T.-F. Yi, Kinetic study on LiFePO4-positive electrode material of lithiumion battery, Ionics 17 (5) (2011) 437-441. 